\title{
Vehicle navigation in populated areas using predictive control with environmental uncertainty handling
}

\author{
KRZYSZTOF SKRZYPCZYK and MARTIN MELLADO
}

\begin{abstract}
This paper addresses the problem of navigating an autonomous vehicle using environmental dynamics prediction. The usefulness of the Game Against Nature formalism adapted to modelling environmental prediction uncertainty is discussed. The possibility of the control law synthesis on the basis of strategies against Nature is presented. The properties and effectiveness of the approach presented are verified by simulations carried out in MATLAB.
\end{abstract}

Key words: motion planning, prediction, uncertainty handling, game theory.

\section{Introduction}

Nowadays more and more mobile robotic solutions are used outdoor. That means robotic vehicles often have to work in populated, dynamic environments under very little human supervision. However coexisting with humans and operating efficiently in such environment, requires a robot must be able to navigate in harmony with traffic participants - humans. Thus the problem of automated navigation in dynamic environments has become an important challenge of contemporary Robotics [1] [3] [5] [8] [9]. In contrast to static and supervised environments, navigating robot in dynamic and uncertain conditions requires many issues to be solved e.g. moving objects detection and tracking, environmental changes prediction, motion planning, and many others. The most common approach to a synthesis of navigation systems which are intended to operate in dynamic conditions is predictive control. Its effectiveness depends on quality of the environmental dynamics model which is created using environment's state observations. Inherent features of each prediction are its inaccuracy and uncertainty. The first one is usually related to a quality of measurement devices or procedures. The second one concerns predictability of the process dynamics. The navigation strategy is calculated using the future, estimated environment's state. In the case the prediction model is ac-

K. Skrzypczyk (corresponding author), is with Institute of Automatic Control, Silesian University of Technology, Akademicka str. 16, 44-100 Gliwice, Poland, e-mail: krzysztof.skrzypczyk@ polsl.pl. M. Mellado is with Instituto de Automática e Informática Industrial Universidad Politecnica de Valencia, Spain, e-mail: martin@ai2.upv.es

This work has been partially supported by BK grant.

Received 9.01.2017. Revised 27.04.2017. 
curate enough and the process is predictable, the navigation plan computed using the prediction usually results in successful, collision free motion even for relatively long time horizon. But in the case when it differs significantly from the real one, the vehicle movement strategy may lead to a collision. Therefore it is very important to provide methods for handling prediction uncertainty in the control process. There are some approaches to uncertainty handling and analysis. The most popular approaches reportet in the literature are: probabilistic approach, fuzzy set based one as well as methods based on Bayesian network formalism [6]. Unfortunately they are quite technically challenging and thus hard to apply in real-world robotic solutions. The key issue in uncertainty robust control is to take action which minimize a risk resulting from a difference between the modeled and the real state of the control process. The technique which allows for relatively easy modelling decision processes uncertainty is Game Theory, in particular Games Against Nature (G.A.N.) [7]. Nature in the G.A.N. formalism symbolizes fictitious player whose behavior cannot be considered as rational. Generally G.A.N. are commonly used in economics, telecommunication or informational sciences. However, this technique can be used also in engineering and technical problems, like control or robots motion planning [10] [11] [4]. Using the game theoretical framework prediction based motion planning can be formulated as a sequence of two-person games in the strategical form. In such games one player is identified with the motion planning system. This system is able to influence the navigation process using decisions (controls) taken from its admissible decision space. The second player is identified with possible to happen, environmental states influencing the process. In this case, these states can by defined as alternative scenarios corresponding to prediction deviations coming from model inaccuracies. By that means uncertainty regarding the prediction can be modeled and involved in the motion control problem. Playing various strategies against such opponent we can obtain the motion plan which takes into account prediction uncertainty. In the remaining part of this paper the motion problem is stated and modeled in the game theoretical framework. Exemplary simulations are presented to illustrate the approach discussed.

\section{Problem overview}

A discussion on uncertainty handling in the predictive navigation of a vehicle is concerned with a system framework presented in Fig.1. An operation idea of this system can be described as follows: The perception subsystem provides information about environment state. The information is assumed to be valuable enough to provide objects' detection and tracking.

In this work none of specific sensory system is considered, but it is assumed it is able to provide information about objects' location. These information generally can be noised and affected by many inaccuracies and they are stored in a data buffer of the size defined. Using these data the objects' motion model is created and a prediction of objects' location is computed in the given time horizon. The aforementioned inaccuracies 


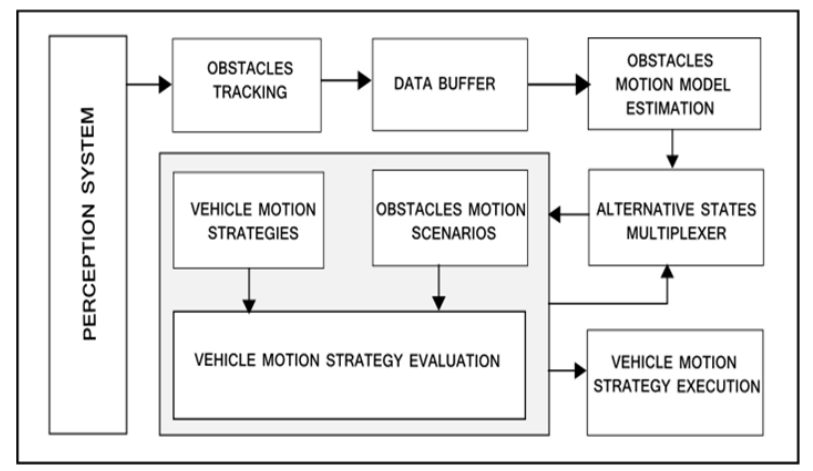

Figure 1: A diagram of the predictive navigation system with uncertainty handling

influence the prediction. This predictions are used for finding the navigation strategy speed and direction of movement which minimize the risk of collision. It is assumed the environment changes prediction is not precise and can differ (even much) from the real state. In order to deal with this fact, alternative scenarios of predicted environmental state are taken into account. These scenarios are input data for the decision making module. In this work the scenarios represent assumed different than identified motion model's parameters. The role of this module is to evaluate particular environmental state - navigation strategies combinations and find the best navigation strategy in the sense of criteria applied. A reasonable navigation strategy should minimize the collision risk in the prediction horizon, on the one hand, and minimize the deviation from the desired movement direction, on the other one.

\subsection{Navigation task formulation}

Usually, navigation problems are decomposed, and analysed as a sequence of simple, short-term, point-to-point navigation ones. The solution of such problem is a sequence of controls that allows the vehicle reaching the desired position defined in the base coordinate frame. In this work the problem is reformulated. From the wheelchair driver perspective the more convenient way for defining the short navigation goal is to follow selected straight-line path instead of reaching an intermediate way point. Let us define the desired path selected in the time $n$ as a tuple:

$$
\Upsilon_{0}(n)=(l(n), \Delta w)
$$

where $l(n)$ is the ray starting at the point $\left(x_{R, n}, y_{R, n}\right)$, angled at $\theta_{R, n}^{*}$. The threshold value $\Delta w$ determines maximal, feasible vehicle distance to the line $l(n)$. The path (1) defines a lane of a width $\Delta w$, the vehicle is supposed to move inside. Thus a primary navigation task can be perceived as a sequence of paths (1) which allows for reaching the destination point. Such an action is more intuitive in the context of a social navigation process. 


\subsection{Problem statement}

$$
R(n)=\left[x_{R, n}, y_{R, n}, \theta_{R, n}\right]^{T}
$$

Let us assume (2) the vehicle is equipped with a sensory system which is able to detect $N$ objects moving in its sensing range and to calculate their positions. Moreover the system is provided with a buffer in which information on objects locations collected in the past can be stored. Assuming the system is a discrete time one with a sampling period $\Delta t$, let us define the set containing locations of objects:

$$
P_{i}=\left\{p_{i, k}\right\}, i=1,2, . ., N, k=\langle n-M, n\rangle
$$

where $p_{i, k}$ is a vector containing coordinates defining location of the $i$ th object in the $k$ th time point. The current time is indexed by $n$ and the number of past observations stored in the buffer is equal to $M$. Using data defined in (3) an estimation of future locations of objects can be found:

$$
\hat{p}_{i, h}^{m}=\bar{p}_{i}^{m}(h), h=n+1,, \ldots, n+H
$$

where $H$ denotes the prediction horizon and $m$ is the model used for calculating the prediction.

Now the short-term navigation task which is the vehicle movement in the desired direction can be stated as follows: In the current time n, using the information on estimated, future objects' location (4), find the control (the direction and velocity of the vehicle):

$$
u_{R}^{*}(n)=\left[\theta_{R}(n), v_{R}(n)\right]^{T}
$$

which applied for $H$ subsequent sampling periods minimize the risk of collision with obstacles and provides the smallest possible deviation from the desired travel direction.

\section{Prediction uncertainty handling}

The vehicle motion strategy is determined using the environmental state prediction (4) and thus highly depends on the quality of this anticipation. Each prediction bears uncertainty and therefore it must be taken into account while planning the motion. In this study we propose to handle prediction uncertainty using game theory framework. Let us consider the process as a two-person game in a strategical form:

$$
G=(D, C)
$$

where $D$ is a decision space of the game while $C$ denotes costs incurred in the aftermath of decisions taken. The decision space is defined as a Cartesian product of the player decision set $D_{G}$ and the decision set $D_{N}$ of a fictitious opponent named Nature [7]. The 
uncertainty considered in this study is represented by various states of Nature specified in the set (7). In turn, each state of Nature represents hypothetical navigation scenario in the presence of circumstances different than predicted in (4). Therefore the uncertainty is handled by considering a number of predictions obtained using various models. Thus the decision set of Nature is defined by:

$$
D_{N}=\{m\}, \quad m \in[1, K]
$$

where $m$ is the model used for calculating the prediction (4). The player's decision set contains admissible controls that can be taken by the vehicle. Let us define this set as:

$$
D_{G}=\left\{u_{R, k}=\left[v_{R, k}, \theta_{R, k}\right]^{T}\right\}=V_{R} \times \Theta_{R}
$$

where:

$$
V_{R}=\left\{v_{R, i}\right\}, i=1, \ldots, \overline{\bar{V}}_{R}, \Theta_{R}=\left\{\theta_{R, j}\right\}, j=1, \ldots, \overline{\bar{\Theta}}_{R}
$$

The set $V_{R}$ contains admissible values of the vehicle velocity. Similarly the set $\Theta_{R}$ contains possible values of the vehicle orientation that can be taken. The motion parameters values defined in (8) correspond to actions usually taken by pedestrians, e.g. marching faster, slower, giving way to an approaching object, stopping etc. Thus, the size of the decision space is not large. A result of applying the control chosen from (8) in the presence of hypothetical scenario distinguished in (7) is evaluated using the function which is to be minimized:

$$
C: D \rightarrow \mathfrak{R}
$$

The values of this function are represented by the matrix $C=\left[c_{i j}\right]$. Rows of this matrix represent admissible controls $i \in D_{G}$, while columns the scenario $j \in D_{N}$ considered. The function (10) modelling process is presented in the next section.

\section{Process modelling}

Let us assume a two component form of the cost evaluation index:

$$
C\left(u_{R, k}, m\right)=\beta_{r} C_{r i s k}^{m}+\beta_{p} C_{p d e v}
$$

where $C_{r i s k}^{m}$ denotes the navigation risk assessment according to the strategy $u_{R, k}$ assuming the $m$ th prediction variant (7). The second component expresses the cost of going off the primary path (1). 


\subsection{Navigation risk evaluation}

In this work the following risk evaluation index is proposed:

$$
C_{r i s k}\left(u_{R, k}, m\right)=\frac{1}{\sum_{h=n+1}^{H} \min _{i=1, \ldots, N}\left|\hat{p}_{R, h}\left(u_{R, k}\right)-\hat{p}_{i, h}^{m}\right|_{L=2}}
$$

This index evaluates the risk of applying the action $u_{R, k} \in D_{G}$ for the time $H$ and is calculated by summarizing distances to the nearest objects. Its value is calculated for the $m$ th prediction variant and is minimized while finding the best control strategy with respect to the collision avoidance aspect. An influence of this component on the index value is tuned experimentally using the weighting factor $\beta_{r}$.

\subsection{Getting off the path cost}

The next aspect of the control $u_{R, k}$ suitability evaluation is calculating the cost related to the path (1) tracking error. Since the vehicle is intended to move inside the lane defined by (1) the suitability of the action is evaluated twofold:

$$
C_{p d e v}\left(u_{R, k}\right)=C_{d i s t}+C_{a n g}
$$

The first component expresses the cost related to getting off the primary path. The second one maps vehicle movement direction change into the cost space. These costs are calculated for the vehicle movement prediction in the time horizon $H$. The first component is determined using the following formula:

$$
C_{d i s t}\left(u_{R, k}\right)=w_{d} \sum_{h=n+1}^{n+H} \hat{d}\left(\hat{p}_{R, h}, l(n)\right)
$$

where $\hat{d}\left(\hat{p}_{R, h}, l(n)\right)$ is the predicted vehicle-path distance calculated in the time point $h$ according to:

$$
\hat{d}\left(\hat{p}_{R, h}, l(n)\right)=\left\{\begin{array}{cc}
\hat{d}\left(\hat{p}_{R, h}, l(n)\right) \text { for } \hat{d}\left(\hat{p}_{R, h}, l(n)\right) \geqslant \Delta w \\
0 & \text { otherwise }
\end{array}\right.
$$

The weighting factor $w_{d}$ is tuned experimentally and is used for balancing the influence of control goals defined by (13). The second component of the cost function is related to the vehicle orientation change resulting from taking the action. This factor is modeled as:

$$
C_{\text {ang }}\left(u_{R, k}\right)=w_{a}\left|\theta_{R, k}-\theta_{R, n}^{*}\right|
$$

The weighting factor $w_{a}$ similarly as in (14) is tuned experimentally. 


\section{Control strategy}

The next stage, after designing and calculating the cost function (11) is to find the best control strategy. This strategy has to take into account various possible environmental scenarios representing states of Nature. Therefore the control for the time horizon $\mathrm{H}$ is determined as the solution of G.A.N. In this study Hurwicz criterion is applied. This criterion characterises an optimistic decision maker, who expects favorable for him circumstances to happen. According to his policy, with some level of optimism $\alpha$, selects a strategy which is the result of the following optimization task:

$$
i_{0}=\min _{i}\left[\alpha \min _{j} c_{i j}+(1-\alpha) \max _{j} c_{i j}\right]
$$

Please note that for $\alpha$ equal to 0 this criterion is equivalent to the pessimistic (Wald) one.

\section{Simulation results}

In order to evaluate the approach considered in this paper, simulation study is carried out. The system functioning is evaluated using a number of scenarios typical for crowded space navigation. In this section an exemplary, very common scenario, is selected as an illustration. The simulated navigation task consists in moving the vehicle along the path set with the given velocity in the presence of moving object. The desired path of the vehicle is marked with the ray outgoing from the point denoting the vehicle position in the time $t=0$. The real path of the object is drawn with circles while the predicted path with dots.

Figure $2 \mathrm{a}$ shows a situation when the vehicle movement strategy is determined using the object movement prediction. In this case the system trusts the model and does not take into account uncertainty of the model. The object changes its movement direction rapidly after the prediction is computed. This situation results in accurate tracking the desired path but leads to collision danger. In a second experiment (Fig. 2b) prediction uncertainty handling mechanism is applied. The vehicle movement parameters are computed using the object movement prediction but with Hurwicz criterion for $\alpha=0$, assuming five various states of Nature. We can see that considering possible to happen scenarios results in much safer vehicle trajectory. This in turn caused the vehicle went off the path much more. The last experiment (fig. 2c) illustrates the same scenario but solved for $\alpha=0.5$. That what can be observed is the tradeoff in the vehicle control. The vehicle gets off the path less, but the distance to the object increases. These experiments show that the method allows to take into account possible environmental changes and adapts the movement strategy to uncertain environmental conditions. 


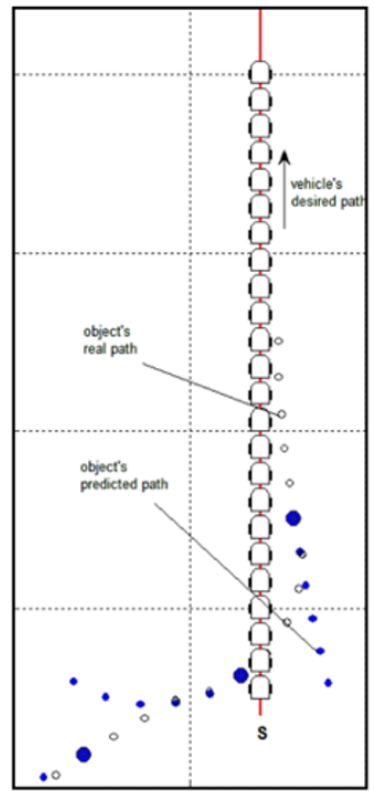

a

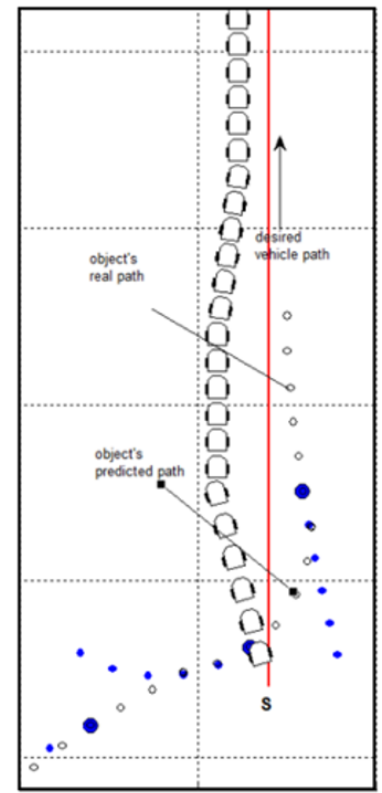

b

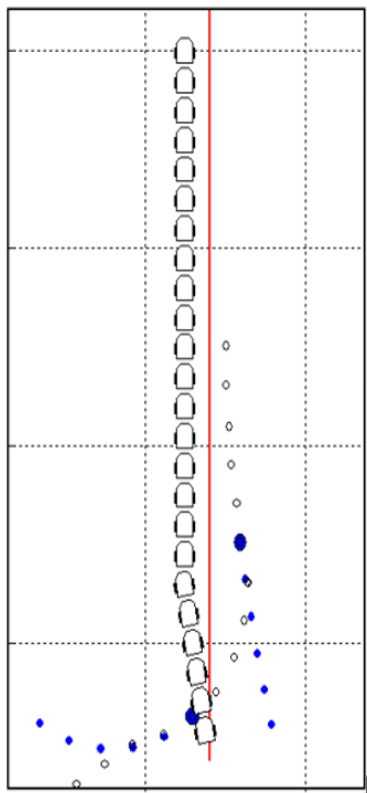

C

Figure 2: Predictive motion planning without uncertainty handling (a), using Hurwicz criterion for $\alpha=0$ (b) and for $\alpha=0.5$ (c)

\section{Conclusions}

In this paper the control system designed for navigating an automated vehicle is discussed. The vehicle is intended to stabilise a rectilinear movement in a direction chosen by the user. Moreover the navigation system is intended to avoid collisions with objects appearing on the course of the vehicle. Since the vehicle is assumed to navigate in populated, crowded spaces the control strategy has to follow the human-aware navigation rules. The main goal of this research is to verify the long term predictive control strategy as a method of generating the socially acceptable movement of the vehicle. The conception presented is simulated using a variety of scenarios. An application of game theory based methods to modelling the uncertainty of prediction model improved robustness of the navigation algorithm. The methodology of the uncertainty handling using G.A.N. seems to be promising and can be applied to many various problems in which the uncertainty modelling play an important role or is indispensable. 


\section{References}

[1] S. Chol, S. Kim and S. OH: Real-time navigation in crowded dynamic environments using Gaussian process motion control. In Proc. of IEEE Int. Conf. on Robotics and Automation, (2014).

[2] D. Ding and R.A. CoOper: Electric-powered wheelchairs: A review of current technology and insight into future directions. IEEE Control Systems Magazine, 25(2), (2005), 22-34.

[3] D. FeIL-SEIFER and M. MATARIC: People-aware navigation for goal-oriented behavior involving a human partner. IEEE Int. Conf. on Development and Learning, Germany, 2 (2011), 24-27.

[4] A. GaluszKa and A. Swierniak: Non-cooperative game approach to multirobot planning. Int. J. of Applied Mathematics and Computer Science, 15(3), (2005), 359-367.

[5] C. GaO, M. SANDS and J.R. Spletzer: Towards autonomous wheelchair systems in urban environments. In Proc. of Int. Conf. on Multisensor Fusion and Integration for Intelligent Systems, Hamburg, Germany, (2012), 77-82.

[6] L.S.DutT and M. KURIAN: Handling of uncertainty - A Survey. Int. J. of Scientific and Research Publications, 3(1), (2013).

[7] J. Milnor: Games Against Nature, in: R. Thrall, C. Coombs and R. Davis (Eds.), Decision Processes, Wiley, New York, 1954, 49-59.

[8] M. Moussaid, D. Helbing and G. Theraulaz: How simple rules determine pedestrian behavior and crowd disasters. Proc. of the National Academy of Science of the United States of America, 108(17), (2011), 6884-6888.

[9] D. Shi, E.G. Collins JR., A. DonATE and X. LiU: Human-aware robot motion planning with velocity constraints. Int. Symp. on Collaborative Technologies and Systems, Irvin, CA, USA, (2008), 490-497.

[10] K. SKRZYPCZYK: Control of a team of mobile robots based on non-cooperative equilibria with partial coordination. Int. J. of Applied Mathematics and Computer Science, 15(1), (2005), 89-97.

[11] K. SKRZYPCZYK: Time optimal tracking a moving target by a mobile vehicle game theoretical approach. Przeglad Elektrotechniczny, 86(3), (2010), 211-215. 